\title{
An assessment of oncology pharmacy educational needs among community pharmacists in Zimbabwe
}

\author{
Maudy Chido Manyau 1, Wilfred Gurupira 2, Dexter Tagwireyi ${ }^{1}$ \\ 1 Department of Pharmacy and Pharmaceutical Sciences, University of Zimbabwe, Zimbabwe \\ 2 University of Zimbabwe Clinical Trials and Research Centre, Zimbabwe
}

\section{Keywords}

Advanced practice

Continuous professional development

Oncology pharmacy

Pharmacy education

\section{Correspondence}

Maudy Chido Manyau

Drug and Toxicology Information Services

Faculty of Medicine and Health Sciences

Parirenyatwa Hospital

Zimbabwe

pinky.manyau@gmail.com

\begin{abstract}
Background: Oncology pharmacy is a specialty that is central to provision and use of anti-cancer medications. In Zimbabwe, pharmacists rely on a Bachelor of Pharmacy degree (B.Pharm.) and in-house training in the provision of oncology services. This study sought to assess knowledge, perceptions, current practices and prior education in oncology pharmacy among community pharmacists. Methods: A cross-sectional survey was carried out among pharmacists working in community pharmacies in Harare. Results: A response rate of $93.0 \%$ was achieved. The mean knowledge score was $4.0 \%$. Up to $96.8 \%$ of pharmacists thought that prior training in oncology was inadequate. Only ten pharmacists in the sample actually dispensed chemotherapy regularly. Of these, none comprehensive measures for the safe handling of hazardous drugs. Conclusion: A B.Pharm. degree does not provide pharmacists with adequate scientific knowledge and competencies required for the provision of oncology pharmacy services. Structured educational programmes for pharmacists in oncology should be implemented.
\end{abstract}

\section{Introduction}

In 2018, Africa contributed $5.8 \%$ to global cancer incidences, and $7.3 \%$ to cancer related deaths (Bray et al., 2018). This high case fatality rate has largely been attributed to the lack of comprehensive cancer care programmes which include treatment, screening and education and training (Jemal et al., 2012). Following the World Health Organisation (WHO) Working Group on National Cancer Control Programmes (NCCPs) meeting held in November 1991 (WHO, 1995), Zimbabwe established a cancer prevention and control committee in 1992. The committee developed a ten year cancer prevention and control strategy, however, it was not fully implemented (MoHCC Zimbabwe, 2015). A second edition of Zimbabwe's NCCP is now available, and one of the objectives of this strategy is the development of a skilled health workforce in oncology (Ministry of Health and Child Care, 2015).

Globally, pharmacy practice is moving towards advanced practice. Internationally, oncology pharmacy has long been recognised as a pharmacy specialty and the scope of oncology pharmacy practice continues to evolve with the changing health needs of cancer patients (Hematology/ Oncology Pharmacy Association, 2019). Many highincome countries are moving towards board certification of oncology pharmacists to ensure that practice is uniform and standards are maintained (Ishikawa, Katz, \& HillBesinque, 2010; Stull, lannucci, \& Bertin, 2006). Standards for specialisation in low-to-middle income countries (LMICS) are determined nationally, and there is no consensus on what these standards should be. Currently, 
there is a heavy reliance on anecdotal information on how pharmacists are providing oncology services in LMICs. This case study sought to provide information on oncology services in a LMIC by assessing oncology pharmacy training and practice in Zimbabwe.

Advanced pharmacy practice in Zimbabwe is still in its infancy. A four year Bachelor of Pharmacy Honours degree (B.Pharm. (Hons)), is the entry level requirement to practice pharmacy in Zimbabwe and much of southern Africa. The B.Pharm. (Hons) programme produces a pharmacist with a general skill-set in pharmaceutical sciences, biomedical and clinical sciences, socio-economic and behavioural. The programme was developed to support the WHO's essential drugs policies, as well as further pharmaceutical care and rational use of medicines (WHO Action Programme on Essential Drugs, 1998). As the healthcare needs of the population change, training needs of pharmacists are also likely to change. In light of the complexity and rapid changes in the field of oncology, it is highly unlikely that a B.Pharm. (Hons) degree is adequate to provide the skill set necessary for the provision of comprehensive quality services to cancer patients.

Review of the University of Zimbabwe B.Pharm. (Hons) curriculum shows that various aspects of oncology pharmacy are taught. These include cancer pathophysiology, anti-cancer drug pharmacology and pharmacotherapeutics. Despite the indication that such aspects are taught, the depth of the content covered cannot be established. For example, the topic 'Neoplasia' is covered under the pathophysiology course. No further detail is provided on the specific cancers, nor their epidemiology and aetiology. The curriculum contains other similar instances. Furthermore, some pharmacists who are in practice have stated that oncology-related content was not covered during undergraduate training, however, others have indicted the opposite. Therefore, the fidelity with which the curriculum has been implemented is unknown.

In Zimbabwe, cancer patients receive their prescriptions from private and public health institutions. The public sector services approximately $80 \%$ of these patients. After receipt of a prescription, the patient or caregiver are tasked with finding the prescribed medications. Oftentimes, oral and intravenous anticancer medicines and supportive treatments are not available in the public sector, and community pharmacies fill the gap. Patients shop around for pharmacies that not only stock the required medicines, but also provide them at costeffective prices. Once medicines have been supplied, patients must return back to the prescribing institution for administration. This makes community pharmacists important in the Zimbabwean oncology treatment pathway, and their inclusion in the multi-disciplinary cancer management teams would facilitate seamless service provision for patients.

In Zimbabwe, community pharmacists who provide oncology pharmacy services regularly require a mixture of competencies of ambulatory clinical pharmacists and specialty pharmacists. This includes in-depth knowledge of oncology disease states, evidence-based treatments, symptom management and supportive care, specialised knowledge of handling of hazardous medications. They must also be proficient in provision of education to patients and caregivers (HOPA, 2019). Beyond provision of drug products, the general scope and quality of oncology services provided by pharmacists in Zimbabwe is unknown.

Inadequate education has been identified as a barrier to quality pharmacy service provision in low-to-middle income countries (Babar \& Scahill, 2014; Smith, 2009). One can hypothesise that if specialised knowledge from the basic B.Pharm. degree is not supplemented, then it is unlikely that the scope nor the quality of oncology-related services meet the expected standards. It is against this background that we sought to assess self-reported sufficiency of prior training, current knowledge, current practices of oncology pharmacy and perceived need for additional training among community pharmacists in Harare. The overarching aim of this study was to identify educational needs of pharmacists in the area of oncology.

\section{Materials and Methods Study Design}

A cross-sectional study was conducted among pharmacists working in registered retail pharmacies in Harare. Randomised quota sampling was used to select a total of 100 pharmacies from a total number of 246 registered pharmacies in Harare. Pharmacies listed on the register of the national medicines regulatory authority, the Medicines Control Authority of Zimbabwe (MCAZ), were stratified according to district within Harare and then a quota for each district determined. Pharmacies were then randomly selected (without replacement) using Microsoft Excel. A study instrument was administered to one pharmacist from each of the selected pharmacies. 


\section{The study instruments}

A coded, anonymous questionnaire was developed to capture participant demographics and information on selfreported sufficiency of prior training, current knowledge, current practices in oncology pharmacy and perceived need for additional training. A combination of Likert scale, closed and open-ended questions were used. The study instrument also included a mini-exam at the end. Information on current practices was only collected for pharmacists who reported that they dispensed cytotoxic medications. The tool was pre-tested on ten pharmacists before being administered to the final study sample.

\section{Ethical considerations}

Approval was obtained from the Pharmacists' Council of Zimbabwe and ethical clearance was sought from University of Zimbabwe College of Health Sciences Joint Research and Ethics Committee (JREC/018) before commencement of the study. Signed informed consent was obtained from all the study participants before the questionnaire was administered.

\section{Data collection and analysis}

The questionnaire was administered by an interviewer after informed consent had been obtained from pharmacists. Data were entered and cleaned in MS Excel 2016, and then imported into Stata (version 13.0) for further analysis. Means, medians and proportions were used to present descriptive statistics. Chi-square tests were used to test for association and logistic regression analysis was used to generate odds ratios (OR). All statistical analysis was performed at a $5 \%$ level of significance.

\section{Results}

Out of 100 pharmacies approached, 93 pharmacists completed the questionnaire. Reasons for non-response were no pharmacist on the premises at the time of the interview $(n=5)$, and refusal to participate $(n=2)$.

\section{Demographic data}

The majority of pharmacists graduated from the University of Zimbabwe or a South African university $(86.0 \%$ and $10.8 \%$ respectively). The remaining pharmacists obtained their degrees from Algeria, Lesotho or Egypt. Demographics are presented in Table I.

\section{Table I: Demographics}

\begin{tabular}{ll}
\hline Variable & $\mathbf{N}=93$ \\
\hline Age, median (IQR) & $33(30-38)$ \\
Males, n (\%) & $47(50.5)$ \\
Country of BPharm attainment, $\mathrm{n}(\%)$ & \\
Zimbabwe & $80(86.0)$ \\
South Africa & $10(10.8)$ \\
Other & $3(3.2)$ \\
Years in Practice, $\mathrm{n}(\%)$ & \\
1-5 & $27(29.0)$ \\
6-10 & $46(49.4)$ \\
11-15 & $10(10.8)$ \\
15+ & $10(10.8)$ \\
Highest Qualification & \\
B.Pharm. (Hons) & $84(90.3)$ \\
Post-graduate diploma & $2(2.2)$ \\
Masters & $7(7.5)$ \\
\hline
\end{tabular}

\section{Prior training}

Over $90 \%$ of interviewed pharmacists stated that they had received theoretical training in handling of (cytotoxic medications and in the aseptic techniques from their undergraduate training (Table II).

\section{Table II: Undergraduate Training}

\begin{tabular}{ll}
\hline Domain & $\begin{array}{l}\text { Received training } \\
\text { Yes, } \mathbf{n} \text { (\%) }\end{array}$ \\
\hline $\begin{array}{l}\text { Theory } \\
\text { Handling of hazardous drugs }\end{array}$ & $89(95.7)$ \\
$\begin{array}{l}\text { Aseptic technique } \\
\text { Oncology therapeutics }\end{array}$ & $92(98.9)$ \\
Practical training & $59(63.4)$ \\
Handling of hazardous drugs & $40(43.0)$ \\
Aseptic technique & $83(89.2)$ \\
Oncology therapeutics & $7(7.5)$ \\
\hline
\end{tabular}

Prior training and perceived training needs were related to where the pharmacists trained.

Pharmacists who learnt in Zimbabwe were more likely to have covered handling of hazardous drugs when compared to those who trained elsewhere $(p<0.001)$. Those who trained in South Africa were more likely to report having been taught oncology therapeutics ( $p<$ 0.001). Furthermore, pharmacists who trained in Zimbabwe were less likely to report that the content covered was adequate OR 0.12 (95\% Cl: 0.03-0.51, $p<$ 0.001).

Less than half of the participants (42.6\%) indicated that training in handling of hazardous drugs was adequate, 


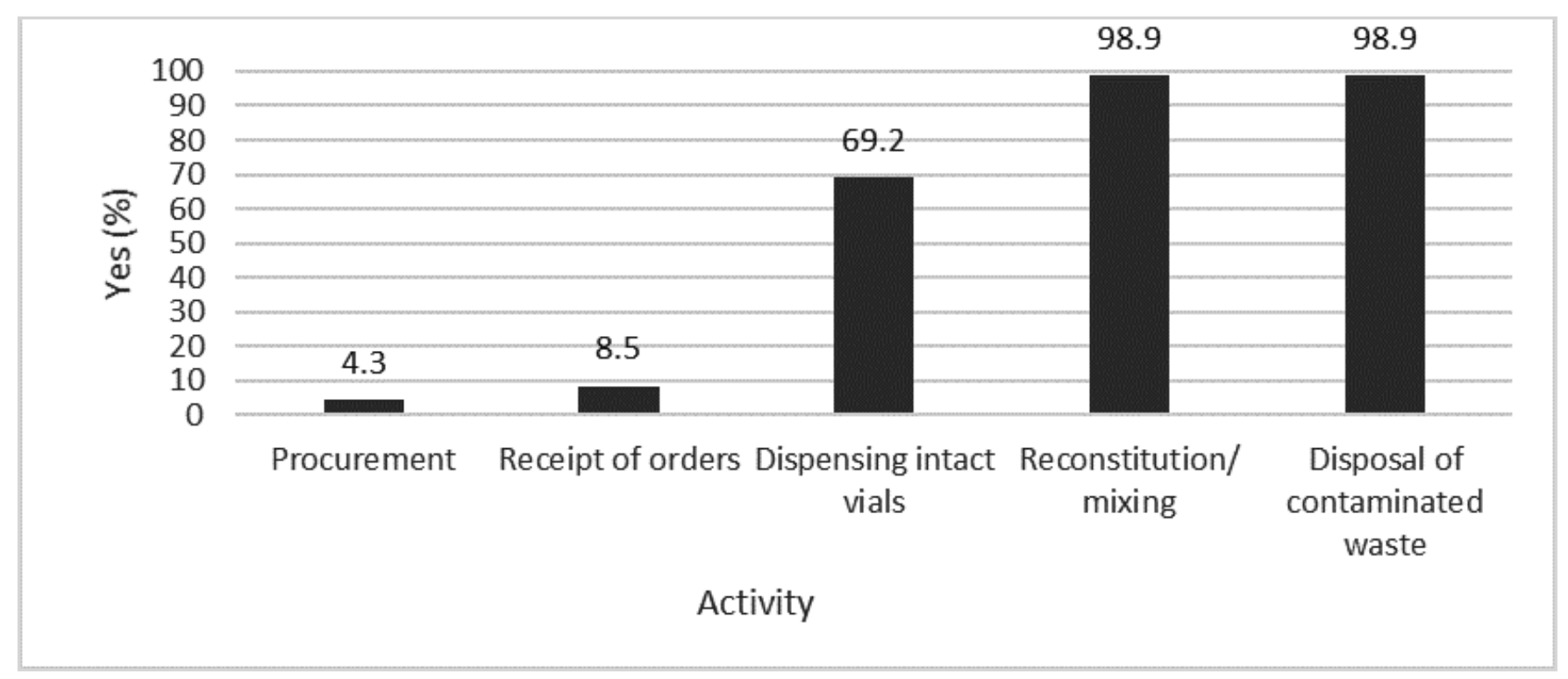

Figure 1: Perceived risk of exposure to cytotoxic medications $(\mathrm{N}=93)$

88.3\% thought that the depth and breadth of aseptic technique taught was sufficient. Lastly, only $7.5 \%$ of participants thought that the training received in oncology therapeutics was sufficient.

\section{Knowledge}

Responses for perceived risk of exposure posed by various activities are displayed in Figure 1.

A mini-exam comprised of 15 questions assessed knowledge of cancer pathophysiology, pharmacology, pharmacy practice and therapeutics. Generally, the marks were less than $50 \%$. The marks ranged from $0(0 \%)$ to 6 $(40.0 \%)$ out of a potential of 15 marks, with a mean of 0.6 (4.0\%). There was no difference in performance according to section. Table III presents the questions asked as well as number of correct responses for the respective questions.

There was a statistically significant difference in mean scores for pharmacists who reported dispensing chemotherapy versus those who did not (1.7 and 0.5 respectively; $p=0.011$ ).

\section{Perceived need for additional training}

92 (98.9\%) of participants agreed that involving pharmacists in cancer patient management would improve outcomes.

Over half of the pharmacists $(58.1 \%)$ were confident to very confident in the provision of chemotherapy related information to prescribers. Only $8.6 \%$ of pharmacists indicated that they were not confident. The majority of
Table III: Mini-exam questions and number of correct responses ( $N=93$ )

\begin{tabular}{ll}
\hline Question & N u m ber \\
of correct \\
responses \\
$n(\%)$
\end{tabular}

Pathophysiology

1.Excluding HIV, which pathogen is Kaposi's 2 (2.2)

sarcoma associated with

2.Which type of genetic mutation commonly 6 (6.5)

causes chronic myeloid leukaemia

Pharmacology

1.What type of receptor is human epidermal $0(0)$ growth factor receptor-2

2. Which chemical class of antineoplastic drugs 2 (2.2) does doxorubicin belong to?

3.Cyclophosphamide is a cell-cycle specific $6(6.5)$ agent $\mathrm{T} / \mathrm{F}$

4.Name 1 hormone modulating drug used for 2 (2.2) prostate cancer

Pharmacy Practice and Pharmacotherapeutics

1.Name any organisation that publishes 4 (4.3)

evidence-based oncology treatment

guidelines

2.What class of biosafety cabinet is acceptable $0(0)$

for reconstituting chemotherapy?

3.State at least 1 standard that chemotherapy 1 (1.1) gloves should comply with?

4.Calculate the BSA for a $1.72 \mathrm{~m} 80 \mathrm{~kg}$ patient $2(2.2)$

5.Which of the following drugs is most likely to 11 (11.8)

cause pulmonary toxicity?

6.Name a drug that raises neutrophil counts 4 (4.3)

7.Which of the following is a cardinal feature of 9 (9.7)

tumour lysis syndrome?

8. Which of the following drugs should be $7(7.5)$

administered using PVC free material?

9.Name a benzodiazepine that is routinely used 1 (1.1)

for anticipatory nausea 


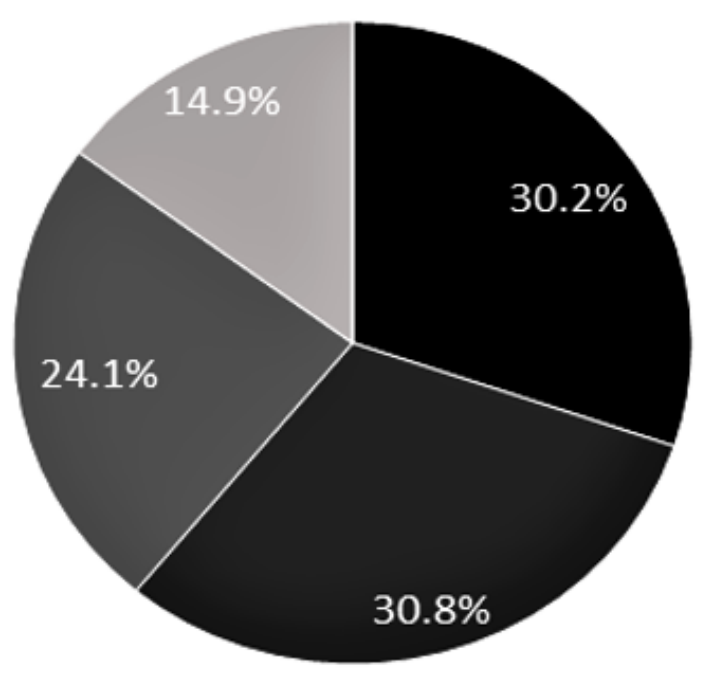

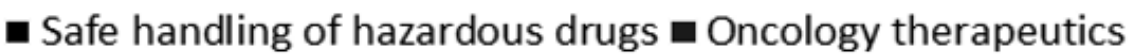
- Pharmacy Practice a Public Health

Figure 2: Educational domains requiring additional training $(N=90)$

pharmacists $(96.8 \%)$ thought that additional training in oncology pharmacy was necessary. Over $60 \%$ felt that additional training should focus on safe handling of cytotoxic medications and oncology therapeutics (Figure 2).

\section{Current practices of pharmacists who provided oncology services}

Current practices were assessed among pharmacists who reported dispensing or stocking cytotoxic medicines regularly. Only ten of the 93 pharmacists indicated that their pharmacies stocked cytotoxic medicines. Of these, eight stated that they did not dispense these medicines often (less than twice a week), and only two dispensed chemotherapy often (three to five days a week).

To assess feasibility of a comprehensive mediation review, pharmacists were asked how often they received chemotherapy prescriptions which highlighted the treatment protocol being followed. Six pharmacists who dispensed chemotherapy reported that chemotherapy prescriptions were accompanied with a treatment protocol less than $30 \%$ of the time. Only four pharmacists indicated that they received treatment protocols most of the time $(70.0 \%$ or more). Six out of ten pharmacists reported that they had never performed medication reviews. Medication reviews was not associated with receipt of a treatment protocol $(p>0.05)$.

Only four pharmacists verified chemotherapy orders. Parameters which were stated to be verified are given below:

- dosage and drug combinations

- pre-medication

- adherence to cycle interval

- checking that the correct drug was being dispensed and expiry date

Six (out of ten) pharmacists indicated that their institutions did not have a policy for handling of cytotoxic medications. All of the four who had handling policies in place stated that cytotoxic drugs were stored separately from other medications in the pharmacy. Only one pharmacist indicated that gloves were used for handling of cytotoxic medications.

Nine pharmacists provided some form of medication counselling at the time of dispensing. All of these respondents stated that they provided information on 
storage of medicines, six provided information on administration and use of medicines. Seven of the ten pharmacists indicated that they counselled on potential side effects.

\section{Discussion}

The main aim of the study was to identify educational needs of Zimbabwean pharmacists in the area of oncology. A high response rate (93.0\%) from a representative sample was obtained. Low test scores and self-reported training needs showed that the basic B.Pharm. (Hons) degree does not adequately prepare pharmacists to provide oncology pharmacy services. Approximately $90 \%$ of the pharmacists had the basic entry level degree (B.Pharm. (Hons)), and they were all obtained from African countries. The large majority (96.8\%) of pharmacists indicated that additional training in oncology pharmacy was necessary in order to provide quality oncology services. This supports prior observations where inadequate education was identified as a barrier to quality pharmacy service provision in low-to-middle income countries (Babar \& Scahill, 2014; Smith, 2009). Oncology pharmacy practice in developing countries is said to lag behind the more developed regions of the world (Strother et al., 2012). This may in part be due the lack of structured training programmes and a heavy reliance on self-driven learning.

In 2001, a workshop to harmonise undergraduate curricula in Southern and Eastern Africa was held in Zimbabwe Kariba (World Health Organization, 2002). As a result, the majority of undergraduate curricula in the region are very similar. Zimbabwean trained pharmacists were overrepresented in the study sample; hence it is difficult to compare competencies across different schools of pharmacy. However, in this study pharmacists who trained in South Africa reported covering more oncologyrelated content. They were also less likely to report inadequacy of the B.Pharm. (Hons) degree. This suggests that although curricula are very similar on paper the depth of the content may differ.

Specialty oncology pharmacies are beginning to emerge in Zimbabwe. These pharmacists working in these pharmacies appear to foster relationships with prescribers. It is very likely that the pharmacists that reported receiving treatment protocols often were resident in these specialist pharmacies. This presents the opportunity for comprehensive pharmaceutical care which includes medication therapy management (MTM). However, pharmaceutical care is premised on scientific knowledge (FIP, 2015). In this case, emphasis on in-depth knowledge of oncology disease states and evidence-based interventions is required (HOPA, 2019). Cancer management in Zimbabwe is further complicated by HIV-infection (Casper, 2011; Chinula, Moses, \& Gopal, 2017). Kaposi sarcoma, cervical cancer and non-Hodgkin's lymphoma are in the top five most frequently diagnosed malignancies (ZNCR, 2016). Cancer patients are a group that is susceptible to polypharmacy, adverse drug reactions and drug-drug interactions (LeBlanc et al., 2015)

With respect to prior training, this study confined assessment to safe handling of cytotoxic medications, aseptic technique and oncology therapeutics. Overall, $63.4 \%$ and $95.7 \%$ of respondents indicated that they had been taught theoretical concepts of oncology therapeutics and safe handling of cytotoxic medications respectively. For practical training, this dropped to as little as $7.5 \%$ for oncology therapeutics and $43.0 \%$ for handling of hazardous drugs. According to Bloom's taxonomy, practical teaching is more likely to engage higher order cognitive activities, and therefore learning, when compared to didactic teaching alone (Bloom, 1956). Furthermore, the conceptual framework by Dreyfus on skill acquisition, indicates that at best, education in a context-free environment produces a novice, competency is only achieved after considerable experience with real situations (Dreyfus \& Dreyfus, 1980). In light of this, the B.Pharm. (Hons) curriculum is not likely to produce competence, neither should it be expected to. Competence, proficiency and expertise will only be attained when learners are solving problems in the practice environment for sustained periods of time (Dreyfus \& Dreyfus, 1980; Katajavuori et al., 2006). This implies that any oncology pharmacy curricula should target post-graduate pharmacists who are in the practice environment.

Several studies have demonstrated the potential mutagenic effects in healthcare workers exposed to cytotoxic drugs (Burgaz et al., 2002; Falck et al., 1979; Nguyen, Theiss, \& Matney, 1982). This prompted development of the initial safe handling of cytotoxic medications standards, and subsequent revisions such as USP 800 and other guidelines (ASHP, 2006; USP 800 / USP, 2019). In this study, only $8.5 \%$ and $69.2 \%$ of participants identified receipt of cytotoxic medications orders and contact with intact vials as activities which carry risk for exposure to cytotoxic medications. Furthermore, none of the pharmacies which stocked hazardous drugs had wellarticulated administrative and engineering controls. Showing that in the absence of structured training, risk assessments will not be conducted properly. Similar 
observations were made by a National Institute for Safety and Health (NIOSH) survey conducted amongst health care professionals working with anticancer drugs. They found that reasons for incorrect handling of cytotoxic medications included lack, or infrequent training of workers (Boiano et al., 2014). This suggests that safe handling of cytotoxic medications is always not intuitive, and training on formulation, implementation and monitoring of comprehensive safety programmes is necessary. Similar findings have been observed in Angolan cancer treatment institutions (da Conceição et al., 2015).

Knowledge attrition was anticipated for pharmacists who did not dispense chemotherapy regularly. The knowledge deficit for pharmacists who regularly dispensed chemotherapy was unexpected. One solution for knowledge gaps in health professionals is continued professional development (CPD). This offers pharmacists the opportunity to maintain and expand their knowledge base and skill set (International Pharmaceutical Federation, 2014). In Zimbabwe, there is a mandatory CPD/ continuing education (CE) point system for annual renewal of practising certificates. The knowledge scores observed in the study suggest that that pharmacists who stocked and dispensed chemotherapy did not complete, or did not retain knowledge from oncology pharmacy related CPD/ CE courses. Additionally, in the absence of metacognition/ or self-awareness, the pharmacist may not select the courses from which they would derive the most benefit. The knowledge scores observed in the study suggest that that pharmacists who stocked and dispensed chemotherapy did not complete, or did not retain knowledge from oncology pharmacy related CPD/ CE courses. One solution for this could be strategic feedback after a CE session. This has been shown to improve metacognition in pharmacy students (Steuber et al., 2017). Additionally, commitment to change statements can also be used to stimulate reflection, guide selection courses and improve commitment to self-development in this niche area (Fjortoft, 2007) . Accreditation of oncology pharmacy $\mathrm{CE}$, which result in recognised competencies may also incentivise pharmacists to develop themselves in oncology.

We also observed the Dunning-Kruger effect in which poor performers showed little self-awareness with respect to their level of expertise (Schlösser et al., 2013). Considering the self-reported inadequacy of prior training and poor test scores, it was paradoxical that approximately half of the pharmacists were confident to very confident in providing cancer therapy related drug information to patients and prescribers. Literature has shown that individuals with a low competence in an area tend to overestimate their capability (Motycka et al., 2010). Hence, self-assessment is not always a valid indication of ability and must be balanced with some form of external assessment. Again, this is a reflection that metacognition in a significant proportion of the group was poor.

There was a positive attitude towards oncology pharmacy as $98.9 \%$ of pharmacists felt that involving pharmacists in cancer patient care would improve patient outcomes. This suggests a willingness of pharmacists to extend their role beyond provision of products. Overall, the findings of this study highlight an opportunity for development of oncology curricula for poorly resourced settings. USAID Academic Model Providing Access to Healthcare (AMPATH) oncology, makes recommendations to train pharmacy personnel in the basics of chemotherapy, safe handling of cytotoxic medications and supportive care (Strother et al., 2012). The current study substantiates these recommendations. Notwithstanding, AMPATH oncology provided pharmacy services through trained pharmacy technicians. In settings like Zimbabwe where a critical mass of pharmacists is available training can be extended to provision of cognitive clinical services.

A limitation of the present was that it only included pharmacists working in the community setting yet the majority of oncology services in Zimbabwe are provided in hospitals. Inclusion of hospital pharmacists would have provided further information on the current status inpatient clinical care, infusion services and ambulatory care. However, this does not invalidate the findings of this study. This is because public hospitals which service approximately $80 \%$ of the population often face stock outages (Ministry of Health and Child Care, 2015), Hence community pharmacists are equally as important as hospital Community pharmacists fill the gap in terms of provision of anticancer medicines and supportive care treatment. Although only $10.0 \%$ of the study sample actually stocked cytotoxic medications, the other $89.2 \%$ could benefit from basic training. This is because supportive care treatments may be supplied by pharmacies that do not actually stock anticancer medicines. Additionally, pharmacy practice in Zimbabwe is not subject to restrictions and there is generally migration of pharmacists across varied areas of practice.

\section{Conclusion}

In conclusion, a basic B.Pharm. (Hons) degree does not provide pharmacists with the skills and competencies required in the provision of oncology pharmacy services. The skills gap may be addressed through structured CPD/ 
CE programmes which are targeted towards pharmacists already providing oncology pharmacy services who are more likely to retain the information.

\section{Availability of data and materials}

Data are available under supplementary information.

\section{Funding information}

This research did not receive any specific grant from funding agencies in the public, commercial, or not-forprofit sectors.

\section{Acknowledgements}

Dr Anna-Mary and facilitators of Health Education Advanced Leadership Program in Zimbabwe (HEALZ) for assistance with refining of the study concept.

\section{Consent for publications}

Not applicable.

\section{Funding}

Not applicable.

\section{Authors' contributions}

MCPM, DT, WTG conception and design. MCPM data collection. MCPM data analysis. MCPM, DT, WTG data interpretation, MCPM, DT, WTG drafting of manuscript. All authors read and approved the final manuscript.

\section{Funding}

The authors received no specific funding for this work.

\section{Competing interests}

No competing interests to declare.

\section{Ethics declaration}

This study was approved by the Pharmacists Council of Zimbabwe, and the University of Zimbabwe Joint Research and Ethics Committee (IRB: JREC/018). Informed consent was obtained from study participants before administration of the questionnaire.

\section{Competing interests}

None to declare.

\section{References}

ASHP. (2006). ASHP Guidelines on handling of hazardous drugs. American Journal of Health System Pharmacy, 63, 1172-1193.

Babar, Z., \& Scahill, S. (2014). Barriers to effective pharmacy practice in low- and middle-income countries. Integrated Pharmacy Research and Practice, 25. https://doi.org/10.2147/IPRP.S35379

Bloom, B.S. (1956). Taxonomy of educational objectives: The classification of educational goals Handbook I, Handbook I,. McKay ; Longman.

Boiano, J.M., Steege, A.L., \& Sweeney, M.H. (2014). Adherence to safe handling guidelines by health care workers who administer antineoplastic drugs. Journal of Occupational and Environmental Hygiene, 11(11), 728-740. PubMed. https://doi.org/ 10.1080/15459624.2014.916809

Bray, F., Ferlay, J., Soerjomataram, I., Siegel, R.L., Torre, L.A., \& Jemal, A. (2018). Global cancer statistics 2018: GLOBOCAN estimates of incidence and mortality worldwide for 36 cancers in 185 countries. CA: A Cancer Journal for Clinicians, 68(6), 394-424. https://doi.org/10.3322/caac. 21492

Burgaz, S., Karahalil, B., Canli, Z., Terzioglu, F., Ançel, G., Anzion, R.B., Bos, R.P., \& Hüttner, E. (2002). Assessment of genotoxic damage in nurses occupationally exposed to antineoplastics by the analysis of chromosomal aberrations. Human \& Experimental Toxicology, 21(3), 129-135. https://doi.org/10.1191/0960327102ht230oa

Casper, C. (2011). The Increasing Burden of HIV-Associated Malignancies in Resource-Limited Regions. Annual Review of Medicine, 62(1), 157-170. https://doi.org/10.1146/annurev-med-050409-103711

Chinula, L., Moses, A., \& Gopal, S. (2017). HIV-associated malignancies in sub-Saharan Africa: progress, challenges, and opportunities. Current opinion in HIV and AIDS, 12(1), 89-95. https://doi.org/10.1097/COH. 0000000000000329

da Conceição, A.V., Bernardo, D., Lopes, L.V., Miguel, F., Bessa, F., Monteiro, F., Santos, C., Oliveira, B., \& Santos, L.L. (2015). Oncology pharmacy units: A safety policy for handling hazardous drugs and related 
waste in low- and middle-income African countries-Angolan experience. Ecancermedicalscience, 9, 575-575. PubMed. https://doi.org/10.3332/ ecancer.2015.575

Dreyfus, S.E., \& Dreyfus, H. (1980). A Five-Stage Model of the Mental Activities Involved in Directed Skill Acquisition. Distribution, 22.

FIP (International Pharmaceutical Federation) (2015). Advanced Practice and Specialisation in Pharmacy: Global Report (online). International Pharmaceutical Federation. Available from: https://www.fip.org/file/ 1397

Fjortoft, N. (2007). The effectiveness of commitment to change statements on improving practice behaviors following continuing pharmacy education. American Journal of Pharmaceutical Education, 71(6), 112. https://doi.org/10.5688/aj7106112

HOPA (Hematology/ Oncology Pharmacy Association). (2019). Further defining the scope of haematology/ oncology pharmacy practice (online). HOPA. Available from: http://www.hoparx.org/images/hopa/resourcelibrary/guidelines-standards/HOPA18_Scope-2_Web2.pdf

International Pharmaceutical Federation. (2014). Continuing Professional Development/Continuing Education in Pharmacy: Global Report (online). International Pharmaceutical Federation (FIP). Available from: https:// www.fip.org/file/1407

Jemal, A., Bray, F., Forman, D., O’Brien, M., Ferlay, J., Center, M., \& Parkin, D.M. (2012). Cancer burden in Africa and opportunities for prevention: Cancer in Africa. Cancer, 118(18), 4372-4384. https://doi.org/10.1002/ cncr. 27410

Ishikawa, K., Katz, M., \& Hill-Besinque, K. (2010). Graduate programs in advanced pharmacy practice in oncology in Japan. American Journal of Pharmaceutical Education, 74(6), Article 111.

Katajavuori, N., Lindblom-Ylänne, S., \& Hirvonen, J. (2006). The Significance of Practical Training in Linking Theoretical Studies with Practice. Higher Education, 51(3), 439-464. https://doi.org/10.1007/ s10734-004-6391-8

LeBlanc, T.W., McNeil, M.J., Kamal, A.H., Currow, D.C., \& Abernethy, A.P. (2015). Polypharmacy in patients with advanced cancer and the role of medication discontinuation. The Lancet Oncology, 16(7), e333-e341. https://doi.org/10.1016/S1470-2045(15)00080-7

Falck, K., Gröhn, P., Sorsa, M., Vainio, H., Heinonen, E., \& Holsti, L.R. (1979). Mutagenicity in urine of nurses handling cytostatic drugs. Lancet (London, England), 1(8128), 1250-1251. https://doi.org/10.1016/ s0140-6736(79)91939-1

Ministry of Health and Child Care. (2015). National cancer prevention and control strategy for Zimbabwe. www.cancerzimbabwe.org

MoHCC Zimbabwe. (2015). Ministry of Health and Child Care: National Cance Prevention and Control Strategy for Zimbabwe 2014-2018. MoHCC.

Motycka, C.A., Rose, R.L., Ried, L.D., \& Brazeau, G. (2010). Selfassessment in pharmacy and health science education and professional practice. American Journal of Pharmaceutical Education, 74(5).

Nguyen, T.V., Theiss, J.C., \& Matney, T.S. (1982). Exposure of Pharmacy Personnel to Mutagenic Antineoplastic Drugs. Cancer Research, 42, 4792-4796.
Stull, D.M., lannucci, A., \& Bertin, R.J. (2006). Board-certified oncology pharmacists: Partners in multidisciplinary care of cancer patients. Community Oncology, 3, 284-286.

Schlösser, T., Dunning, D., Johnson, K.L., \& Kruger, J. (2013). How unaware are the unskilled? Empirical tests of the "signal extraction" counterexplanation for the Dunning-Kruger effect in self-evaluation of performance. Journal of Economic Psychology, 39, 85-100. https:// doi.org/10.1016/j.joep.2013.07.004

Smith, F. (2009). The quality of private pharmacy services in low and middle-income countries: A systematic review. Pharmacy World \& Science, 31(3), 351-361. https://doi.org/10.1007/s11096-009-9294-z

Steuber, T.D., Janzen, K.M., Walton, A.M., \& Nisly, S.A. (2017). Assessment of Learner Metacognition in a Professional Pharmacy Elective Course. American Journal of Pharmaceutical Education, 81(10), 6034. https://doi.org/10.5688/ajpe6034

Strother, R.M., Rao, K.V., Gregory, K.M., Jakait, B., Busakhala, N., Schellhase, E., Pastakia, S., Krzyzanowska, M., \& Loehrer, P.J. (2012). The oncology pharmacy in cancer care delivery in a resource-constrained setting in western Kenya. Journal of Oncology Pharmacy Practice, 18(4), 406-416. https://doi.org/10.1177/1078155211434852

USP 800 / USP. (2019). Available from: https://www.usp.org/ compounding/general-chapter-hazardous-drugs-handling-healthcare

WHO (World Health Organisation) (1995). National Cancer Control Programmes: Policies and material guidelines.

WHO Action Programme on Essential Drugs. (1998). Revision of undergraduate pharmacy curricula: Report on an informal consultation in Nyanga, Zimbabwe, 18-20 April 1997. WHO/DAP/98.1. WHO IRIS. https://apps.who.int/iris/handle/10665/63940

World Health Organization. (2002). Harmonization of undergraduate pharmacy curricula in southern and eastern Africa: Future trends: Report of a workshop in Kariba, Zimbabwe 8-11 April 2001. WHO/EDM/PAR/ 2002.4. WHO IRIS. https://apps.who.int/iris/handle/10665/67717

ZNCR, M. (2016). Zimbabwe National Registry Cancer Regisry: 2016 Annual Report. ZNCR. 